\title{
Effects of flutter and PEP mask physiotherapy on symptoms and lung function in children with cystic fibrosis
}

\author{
C.M.Q. van Winden*+, A. Visser**, W. Hop , P.J. Sterk+, S. Beckers*, J.C. de Jongste*
}

Effects of flutter and PEP mask physiotherapy on symptoms and lung function in children with cystic fibrosis. C.M.Q. van Winden, A. Visser, W. Hop, P.J. Sterk, S. Beckers, J.C. de Jongste. CERS Journals Ltd 1998.

ABSTRACT: Recently, the flutter was introduced as a new device to improve sputum expectoration. Preliminary data suggested a significant improvement in expectoration and lung function during flutter treatment in patients with cystic fibrosis (CF). The aim of the present study was to compare the effects of the flutter and the positive expiratory pressure (PEP) mask on symptoms and lung function in children with CF.

In a crossover randomized study 22 patients with $C F$ (mean age 12 yrs, range 7-17 yrs) performed physiotherapy using either the flutter or the PEP mask twice a day during two treatment periods of 2 weeks, separated by a one week wash-out period, in a random sequence. Lung function parameters (peak expiratory flow, forced vital capacity (FVC), forced expiratory volume in one second, maximal midexpiratory flow, maximal expiratory flow at $25 \%$ of $\mathrm{FVC}$, thoracic gas volume, total lung capacity, residual volume/total lung capacity, airway resistance and specific airway conductance) and changes in transcutaneous oxygen haemoglobin saturation were assessed before and after the first supervised session and at the end of each treatment period. Throughout the study peak flow was measured and symptoms were scored daily.

No significant changes in any lung function parameter occurred after a single session or after 2 weeks of physiotherapy with either method. There was no difference in acceptability and subjective efficacy.

In conclusion, any superiority of the flutter over the positive expiratory pressure mask technique for expectoration could not be confirmed during 2 weeks of daily treatment in children with cystic fibrosis. Both methods are well accepted by children and do not change lung function. Long-term comparison of both methods, including expectoration measurements, seems to be required for further evaluation of the potential success of physiotherapy in cystic fibrosis. Eur Respir J 1998; 12: 143-147.
Depts of *Pediatrics, Division of Pediatric Respiratory Medicine, **Physiotherapy and Biostatistics, Erasmus University and University Hospital/Sophia Children's Hospital Rotterdam, The Netherlands. +Dept of Pulmonology, Lung Function Laboratory, Leiden University Medical Centre, The Netherlands.

Correspondence: J.C. de Jongste

Dept of Pediatrics

Division of Pediatric Respiratory Medicine

Sophia Children's Hospital

Dr Molewaterplein 60

3015 GJ Rotterdam

The Netherlands

Fax: 31104636801

Keywords: Cystic fibrosis

expectorations

flutter

lung function

physiotherapy

positive expiratory pressure mask

Received: June 301997

Accepted after revision January 71998

Presented as a poster at the XIIth Annual Cystic Fibrosis Congress in Jerusalem, June 1996; an abstract appeared in Israel J Med Sci 1996; 32: S275.
Cystic fibrosis (CF) patients have viscous bronchial secretions, endobronchial infection and, hence, destruction of the lung and deterioration of lung function [1]. Sputum expectoration, aided by chest physiotherapy (CPT), postural drainage, percussion and coughing, have been standard therapy for many years $[1,2]$. Self-administered physiotherapy techniques, including the forced expiration technique (FET), active cycle of breathing technique (ACBT), autogenic drainage and the use of a positive expiratory pressure (PEP) mask provide personal independence for the patient and have largely replaced earlier techniques [3-11]. It is uncertain whether any of these newer methods, or combinations of different techniques, are better than others [1, 11-14].

A new device to facilitate clearance of mucus is the flutter. When the patient exhales through the flutter, oscillations of expiratory pressure and airflow vibrate the airway walls, should diminish adhesiveness of mucus, decrease the collapsibility of the airways and accelerate airflow [15]. The use of the flutter may therefore result in expectoration of a greater amount of sputum than the use of postural drainage or coughing in CF patients [15]. Also, improvement in lung function after 14 days of physiotherapy with the use of the flutter was reported in adult patients with chronic obstructive bronchitis [16] and preliminary data suggest the same for patients with CF [17, 18]. In contrast, most studies with the PEP mask failed to show short-term benefit or improvement in lung function or symptoms $[5,19,20]$ or were inconclusive because other aspects of the treatment may have been responsible for an observed effect [21]. It seems, therefore, that the flutter might have advantages over the more commonly used PEP mask. This study aimed to compare the effects of the flutter and the PEP mask in children with CF.

Materials and methods

\section{Patients}

Twenty-two patients, 12 males and 10 females, with CF confirmed by sweat test and/or deoxyribonucleic acid (DNA) mutation analysis, with a mean age of $12 \mathrm{yrs}$ 
(range 7-17 yrs) participated in the study. Characteristics of these subjects are given in table 1 . All patients were clinically stable for at least 2 weeks prior to the study, as shown by stable peak flows and symptom scores by questionnaire, could perform lung function tests reproducibly (within-subject range of three successive forced expiratory volume in one second (FEV1) manoeuvres <10\%) and had productive cough on most days. All participants gave written informed consent.

\section{Study design}

The study has a crossover design (fig. 1). Patients continued to receive their usual medication throughout the study (excluded: mucolytics other than saline) in unchanged doses and they continued FET. Peak flow was measured daily after the morning physiotherapy session by means of a Mini-Wright peak-flow meter (Clement Clarke, London, UK) and a questionnaire on symptoms of daytime and night-time cough, sputum production and shortness of breath (each being scored on a three-point scale) was completed daily. The cumulative number of symptoms in a given period was taken as the symptom score.

Each patient was assigned randomly to one of two treatment sequences. After a one-week run-in period (first wash-out), either the PEP mask or the flutter was introduced and continued for 2 weeks, twice a day. After a second wash-out period of 1 week, both groups changed to the alternative treatment by flutter or PEP mask for

Table 1. - Baseline characteristics of cystic fibrosis patients

\begin{tabular}{|c|c|c|c|}
\hline & $\begin{array}{l}\text { Total group } \\
\mathrm{n}=22\end{array}$ & $\begin{array}{l}\text { PEP first } \\
\mathrm{n}=10\end{array}$ & $\begin{array}{l}\text { Flutter first } \\
n=12\end{array}$ \\
\hline Female $n$ & 10 & 6 & 4 \\
\hline Age yrs* & $12(7-17)$ & $13(8-16)$ & $11(7-17)$ \\
\hline $\mathrm{FEV}_{1} *_{+}$ & $82(55-129)$ & $79(63-120)$ & $86(55-129)$ \\
\hline $\mathrm{FVC}^{*+}$ & $95(77-133)$ & $90(77-126)$ & $99(79-133)$ \\
\hline Inhaled $\beta$-agonists & 15 & 7 & 8 \\
\hline Inhaled corticosteroids & 7 & 4 & 3 \\
\hline Inhaled antibiotics & 1 & 1 & 0 \\
\hline Oral antibiotics, daily & 14 & 7 & 7 \\
\hline Oral corticosteroids & 3 & 2 & 1 \\
\hline $\begin{array}{l}\text { Pseudomonas coloniza- } \\
\text { tion }\end{array}$ & 14 & 7 & 7 \\
\hline
\end{tabular}

*: median (range). +: lung function on study entry, expressed in percentage predicted values [22]. PEP: positive expiratory pressure; FEV1: forced expiratory volume in one second; FVC: forced vital capacity.

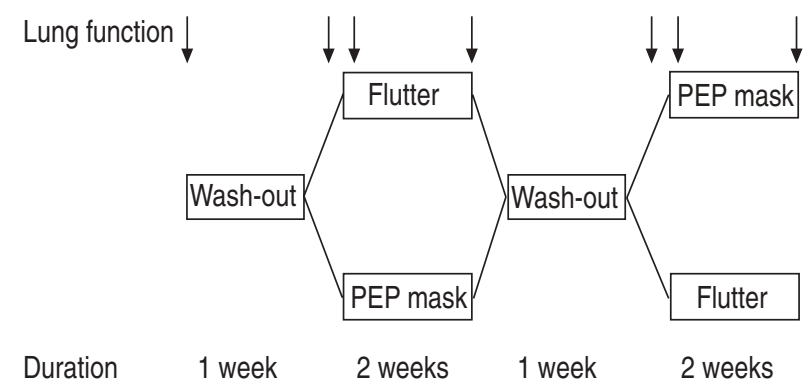

Fig. 1. - Study design. PEP: positive expiratory pressure. another 2 weeks. During the two wash-out periods, all patients performed routine physiotherapy with FET, preceded by nebulization of $10 \mathrm{~mL}$ of $0.9 \% \mathrm{NaCl}$, but without the PEP mask or the flutter. At the end of each treatment period the patients and their parents completed a questionnaire regarding the acceptability and perceived efficacy of each physiotherapy method. At the end of the study the patients were asked for their preference. The study was approved by the Medical Ethics Committee of the hospital and was performed according to the directives of Good Clinical Practice.

\section{Physiotherapy regimens}

Ten millilitres of $0.9 \% \mathrm{NaCl}$ was nebulized via an ultrasonic nebulizer (Medasonic; Medasto, Woerden, The Netherlands) before each physiotherapy session. All patients were familiar with the use of the PEP mask and had used this at home for an average of 4.0 yrs (range 0.5-8 yrs) before the study. All patients were instructed how to use the flutter and the PEP mask on two occasions preceding each treatment period. After each treatment period the technique was checked by observing and scoring various aspects of the entire procedure. A paediatric physiotherapist experienced in CF treatment (A.Visser) supervised the instruction and the use of the PEP mask and the flutter.

Both techniques were combined with FET to clear the central airways. The number of huff and cough manoeuvres was standardized, because it has been shown that huffing and coughing as individual techniques are effective as well $[12,23,24]$.

\section{Positive expiratory pressure mask}

The PEP-mask (Astra Meditec, Rijswijk, The Netherlands) was used as follows. Sitting with a straight back and the elbows supported on the table, the patient pressed the mask against the face with both hands. After a quiet inhalation, a slow active exhalation followed. The exhalation was restricted by a resistance selected to cause a positive expiratory pressure of $8-12 \mathrm{cmH}_{2} \mathrm{O}$. The patient exhaled 15 times through the mask. Thereafter, the patient huffed three times and evacuated sputum by a voluntary cough. This sequence was repeated five times [25, 26].

\section{Flutter}

The flutter (VarioRaw, Aubonne, Switzerland) was used as follows. Sitting with a straight back and the elbows supported on the table, the patient held the flutter horizontally. The patient inhaled deeply, held their breath for 2 or $3 \mathrm{~s}$ and then took the mouthpiece into the mouth and exhaled quietly yet quickly enough to activate the flutter. The patient had to keep their cheeks as stiff as possible. This procedure was repeated 15 times, following which the patient huffed three times and evacuated sputum by a voluntary cough. This sequence was repeated five times. The patient obtained the maximal vibration sensation by tilting the flutter upwards or downwards by a few degrees. 


\section{Lung function}

On the first day of the run-in period, flow-volume curves were performed to establish baseline values of dynamic lung volumes and flows. Lung function was also measured on the first day of each of the two treatment periods before and $30 \mathrm{~min}$ after the first supervised session of physiotherapy. At the end of 14-day treatment period, lung function parameters were measured $30 \mathrm{~min}$ after physiotherapy. Prior to lung function tests, the patients discontinued any inhalation therapy except saline for 8 h. Peak expiratory flow (PEF), forced vital capacity (FVC), FEV1, maximal expiratory flow at $50 \%$ of FVC (MMEF) and maximal expiratory flow at $25 \%$ of FVC (MEF25) were measured by means of a calibrated pneumotachograph (Masterlab, Jaeger, Würzburg, Germany). The best of three forced expiration measurements was taken. Total lung capacity (TLC), residual volume (RV/ TLC), airway resistance (Raw) and specific airway conductance ( $\mathrm{s} G$ aw) were measured by means of whole body plethysmography (Masterlab, Jaeger). The results were expressed as a percentage of predicted values [22]. After the first session of physiotherapy and after 2 weeks of treatment the effect of a bronchodilator (terbutaline $1 \mathrm{mg}$ via a Turbuhaler) was measured by repeating the flow-volume curve 30 min after treatment. The percentage of transcutaneous haemoglobin oxygen saturation $\left(\mathrm{Stc}, \mathrm{O}_{2}\right)$ was measured by pulse oximetry (Ohmeda Biox, Louisville $\mathrm{CO}$, USA) before, during and $30 \mathrm{~min}$ after all supervised sessions.

\section{Statistical analysis}

The results of the lung function tests before and after the first session of physiotherapy following the wash-out periods and changes in lung function parameters before and after 2 weeks of the two different physiotherapy methods were compared by means of paired t-tests. The appropriateness of this test was verified by securing that there were no carry-over or period effects using methods which are suitable for crossover studies [27]. In view of the number of parameters compared, statistical significance was assumed at $\mathrm{p}=0.01$ (two-sided). With $n=22$, the power to detect differences of roughly $1 \mathrm{SD}$ at $\mathrm{p}=0.01$ is at least $80 \%$. Data given are means \pm SEM unless indicated otherwise.

\section{Results}

All 22 patients completed the study. Twelve patients started to use the flutter and 10 started to use the PEP mask in the first treatment period. At the onset of the study the mean results of flow-volume measurements, peak flow values and symptom scores were similar between both groups and did not change significantly during the run-in period. The lung function parameters measured before physiotherapy on the first day of each twoweek treatment period were also similar (table 2). The mean symptom score was $10 \pm 1$ during the first and $10 \pm 2$ during the second wash-out period. The mean PEF was $309 \pm 15 \mathrm{~L} \cdot \mathrm{min}^{-1}$ during the first and $319 \pm 20 \mathrm{~L} \cdot \mathrm{min}^{-1}$ during the second wash-out period (nonsignificant difference, Ns).
Table 2. - Baseline characteristics of cystic fibrosis patients

\begin{tabular}{|c|c|c|c|c|c|c|}
\hline & \multicolumn{3}{|c|}{ Flutter } & \multicolumn{3}{|c|}{ PEP mask } \\
\hline & Before & After & $\begin{array}{l}\text { After } 2 \\
\text { weekss }\end{array}$ & Before & After & $\begin{array}{l}\text { After } 2 \\
\text { weeks }\end{array}$ \\
\hline FVC & $99 \pm 4$ & $99 \pm 4$ & $99 \pm 4$ & $95 \pm 3$ & $96 \pm 3$ & $97 \pm 3$ \\
\hline $\mathrm{FEV}_{1}$ & $87 \pm 5$ & $86 \pm 5$ & $88 \pm 4$ & $85 \pm 4$ & $86 \pm 4$ & $86 \pm 4$ \\
\hline$\Delta \mathrm{FEV}_{1} *$ & & $+5 \pm 1$ & $+5 \pm 1$ & & $+6 \pm 1$ & $+6 \pm 1$ \\
\hline$\Delta \mathrm{FEV}_{1 / \mathrm{FVC}^{*}}$ & & $+3 \pm 1$ & $+2 \pm 1$ & & $+2 \pm 1$ & $+3 \pm 1$ \\
\hline TGV & $120 \pm 5$ & $119 \pm 4$ & $122 \pm 4$ & $118 \pm 3$ & $120 \pm 3$ & $121 \pm 4$ \\
\hline TLC & $106 \pm 3$ & $107 \pm 2$ & $105 \pm 3$ & $106 \pm 3$ & $107 \pm 3$ & $107 \pm 2$ \\
\hline RV/TLC & $139 \pm 7$ & $140 \pm 7$ & $138 \pm 8$ & $140 \pm 7$ & $142 \pm 8$ & $145 \pm 7$ \\
\hline PEF & $92 \pm 3$ & $90 \pm 3$ & $92 \pm 3$ & $92 \pm 3$ & $91 \pm 2$ & $90 \pm 2$ \\
\hline MEF25 & $41 \pm 5$ & $38 \pm 4$ & $43 \pm 5$ & $41 \pm 5$ & $44 \pm 6$ & $42 \pm 5$ \\
\hline MMEF25-75 & $55 \pm 5$ & $57 \pm 6$ & $54 \pm 5$ & $54 \pm 5$ & $53 \pm 5$ & $55 \pm 5$ \\
\hline Raw & $237 \pm 22$ & $239 \pm 22$ & $240 \pm 20$ & $234 \pm 17$ & $239 \pm 20$ & $246 \pm 20$ \\
\hline
\end{tabular}

All values are expressed as a percentage \pm 1 SEM of normal predicted, according to ZapLetal et al. [22]. *: effect of trebutaline on forced expiratory volume in one second (FEV1) and FEV $1 /$ forced vital capacity (FVC), expressed as \% change. TGV: thoracic gas volume; TLC: total lung capacity; RV: residual volume; PEF: peak expiratory flow; MEF25: maximal mid-expiratory flow at 25\% of FVC; MMEF25-75: maximal midexpiratory flow; Raw: airway resistance.

These figures were similar for each of the two sequence groups. During the PEP periods the mean peak flow was similar to that during the flutter periods $\left(316 \mathrm{~L} \cdot \mathrm{min}^{-1}\right.$ and $319 \mathrm{~L} \cdot \mathrm{min}^{-1}$, respectively, Ns). Slightly more symptoms were recorded during the PEP periods than during the flutter periods, not due to a specific symptom category (average symptom scores 23 and 19, respectively, ns). The results of the lung function tests are shown in table 2 . There were no significant changes in mean values of any of the lung function parameters after one session or after 2 weeks of physiotherapy with both methods. The effect of inhaled terbutaline after 2 weeks of physiotherapy with either method was unchanged from the effect on the first day.

It was postulated that those patients preferring one of the devices could have shown more improvement with that device than with the other. Therefore, data were analysed separately with the patients grouped according to their preference. However, those who preferred the PEP mask were similar to those preferring the flutter in all respects.

The mean $\mathrm{Stc}, \mathrm{O}_{2}$ before the sessions with the PEP mask was $96 \pm 0.2 \%$ (range 94-99\%). No significant differences were found between the two treatments before, directly after and 30 min after physiotherapy. During and immediately after the physiotherapy sessions desaturation (arterial oxygen saturation $\left.\left(\mathrm{Sa}, \mathrm{O}_{2}\right)<92 \%\right)$ occurred in one patient using the PEP mask and in six patients using the flutter. This lasted for $<2 \mathrm{~min}$ in all but one patient.

Evaluation of the correct use of the PEP mask and the flutter after each treatment period showed good compliance and good technique of performance, with the exception of breath holding with the use of the flutter: 21 of 22 patients held their breath after inhalation for $<3 \mathrm{~s}$.

Subjective improvement, preference, time needed and effect on sputum production were similar for both treatments. Ten patients preferred the PEP mask, 11 patients preferred the flutter and one patient had no preference. 


\section{Side-effects}

Five patients complained of dizziness as a result of the use of the flutter. This improved after they were instructed not to breathe too quickly or too forcefully through the flutter.

\section{Discussion}

This study compared two physiotherapy aids, the flutter and the PEP mask, in children with CF. There was no significant change in lung function after one session or after 2 weeks of twice-daily physiotherapy with the flutter or the PEP mask. Oxygenation and symptoms were similar during treatment with both methods.

It needs to be explained that sputum production, a logical outcome variable for expectoration studies [15, 28], was not taken into account because, in our and others' experience, it appears that children will swallow most sputum and mixing with saliva will occur [7, 12, 29]. Several studies have tried to measure lung function changes as a result of expectoration methods, some with negative results [5, 19] and some with significant changes even after a single session [11]. Widely varying methodologies in patients with varying severity of lung disease could well explain the different outcomes. Regarding the PEP mask, several studies failed to show an improvement in forced expiratory flows or lung volumes after several weeks of daily treatment $[5,20]$. In contrast, others showed a significant improvement in lung volumes and expiratory flows after 15 days of PEP mask physiotherapy by $\mathrm{CF}$ patients hospitalized for a pulmonary exacerbation [21]. It is unclear, however, whether improvements in the latter study were the result of physiotherapy or of another aspect of treatment. A preliminary report described a significant im-provement in Raw after one session of physiotherapy with the PEP mask [17]. Some authors have used "highpressure PEP" $[11,21]$, by combining forced expiration and the PEP mask. It may not be appropriate to compare the present results, obtained with low-pressure PEP, with those of high-pressure PEP, although there is no evidence that high and low PEP have different effects.

In agreement with two other studies [30, 31], no significant change in lung function was seen after one session of physiotherapy with the flutter. Other authors, however, have reported a significant improvement in lung function after one session with the flutter and an effect of the flutter on end-expiratory flows $[17,18]$. However, as these patients were hospitalized and received antibiotics, valid estimation of the separate effect of physiotherapy seems impossible. Improvement in lung function (VC, FEV1 and PEF) after 14 days of physiotherapy with the flutter was demonstrated in elderly patients with chronic obstructive pulmonary disease [16].

As a result of physiotherapy lung volumes may increase and a reduction in unequal ventilation may lead to an improvement in gas exchange and, hence, oxygenation. However, in agreement with others $[31,32]$, we found no significant changes in oxygenation with the use of the PEP mask or the flutter. This could be due to the relatively high baseline values of our patients; indeed, a significant improvement in saturation during use of the PEP mask was documented in a group of $\mathrm{CF}$ patients with much lower baseline values [33]. However, six subjects had short-lasting desaturations after the flutter and this was seen in only one patient after PEP. It may be speculated that such short desaturations reflect a transient mismatch of ventilation and perfusion, owing to the mobilization of sputum from peripheral airways, and the data suggest that this happens more often with the flutter.

As a group, the patients did not prefer one of the two devices. However, some younger patients apparently had difficulty in using the flutter correctly. This differs from experience reported in other studies that physiotherapy with the flutter can be performed by young children from the age of 3 yrs [28]. The patients in the present study had performed physiotherapy with the use of the PEP mask for an average of 4.0 yrs prior to the study, while the flutter was a new device for them. This may have influenced the results but this seems unlikely, as good compliance was observed with both techniques.

In conclusion, the effects of two physiotherapy techniques, the positive expiratory pressure mask and the flutter, on symptoms and lung function were compared in children with cystic fibrosis. No differences were found between the techniques. Long-term studies may reveal effects that are not apparent after 2 weeks. For the time being, it seems best to choose the method that matches the patient's abilities and preference in order to improve compliance with the physiotherapy regimen.

Acknowledgements: The authors are grateful to J.H. Bischofsberger from VarioRaw, Aubonne, Switzerland, for providing the flutters.

\section{References}

1. Zach MS. Lung disease in cystic fibrosis: an updated concept. Pediatr Pulmonol 1990; 8: 188-202.

2. Desmond KJ, Schwenk WF, Thomas E, Beaudry PH, Coates AL. Immediate and long-term effects of chest physiotherapy in patients with cystic fibrosis. $J$ Pediatr 1983; 103: 538-542.

3. Bauer ML, McDougal J, Schoumacher RA. Comparison of manual and mechanical chest percussion in hospitalized patients with cystic fibrosis. J Pediatr 1994; 124 : 250-254.

4. Cerny FJ. Relative effects of bronchial drainage and exercise for in-hospital care of patients with cystic fibrosis. Phys Ther 1989; 69: 633-639.

5. Tyrrell JC, Hiller EJ, Martin J. Face mask physiotherapy in cystic fibrosis. Arch Dis Child 1986; 61: 598-611.

6. Sutton PP, Parker RA, Webber BA, et al. Assessment of the forced expiration technique, postural drainage and directed coughing in chest physiotherapy. Eur J Respir Dis 1983; 64: 62-68.

7. Prasad SA. Current concepts in physiotherapy. $J R$ Soc Med 1993; 86: 23-29.

8. Miller S, Hall DO, Clayton CB, Nelson R. Chest physiotherapy in cystic fibrosis: a comparative study of autogenic drainage and the active cycle of breathing techniques with postural drainage. Thorax 1995; 50: 165-169.

9. Tønnesen P, Stovring S. Positive expiratory pressure (PEP) as lung physiotherapy in cystic fibrosis: a pilot study. Eur J Respir Dis 1984; 65: 419-422.

10. Van Hengstum M, Festen J, Beurskens C, Hankel M, Beekman F, Corstens F. Conventional physiotherapy and forced expiration manoeuvres have similar effects on tracheobronchial clearance. Eur Respir J 1988; 1: 758-761. 
11. Pfleger A, Theissl B, Oberwaldner B, Zach MS. Self-administered chest physiotherapy in cystic fibrosis: a comparative study of high-pressure PEP and autogenic drainage. Lung 1992; 170: 323-330.

12. Thomas J, Cook CJ, Brooks D. Chest physical therapy management of patients with cystic fibrosis. A meta-analysis. Am J Respir Crit Care Med 1995; 151: 846-850.

13. Corey M, Levison H, Crozier D. Five- to seven-year course of pulmonary function in cystic fibrosis. Am Rev Respir Dis 1976; 114: 1085-1092.

14. Fick RB, Stillwell PC. Controversies in the management of pulmonary disease due to cystic fibrosis. Chest 1989; 95: 1319-1327.

15. Konstan MW, Stem RC, Doershuk CF. Efficacy of the flutter device for airway mucus clearance in patients with cystic fibrosis. J Pediatr 1994; 124: 689-693.

16. Cegla UH, Ems B. Physiotherapy and chronic obstructive bronchitis, experiments with the VRP1 desitin. Pneumologie 1993; 47: 636-639.

17. Casaulta Aebischer C, Frey U, Schibler A, Kraemer R. Efficacy of chest physiotherapy (CPT) (PEP mask versus flutter) in patients with cystic fibrosis (CF). Eur Respir $J$ 1993; 6: 220S.

18. Althaus P, Leuenberger P.H. Comparison of the PEPmask and the flutter in the bronchial hygiene of cystic fibrosis patients. Schweiz Med Wochenschr 1989; 119: 39.

19. Braggion C, Cappelletti LM, Cornacchia M, Zanolla L, Mastella G. Short-term effects of three chest physiotherapy regimens in patients hospitalized for pulmonary exacerbations of cystic fibrosis: a cross-over randomized study. Pediatr Pulmonol 1995; 19: 16-22.

20. Steen HJ, Redmond AOB, O'Neill D, Beattie F. Evaluation of the PEP mask in cystic fibrosis. Acta Paediatr Scand 1991; 80: 51-56.

21. Oberwaldner B, Theissl B, Rucker A, Zach MS. Chest physiotherapy in hospitalized patients with cystic fibrosis: a study of lung function effects and sputum production. Eur Respir J 1991; 4: 152-158.
22. Zapletal A, Samének M, Paul T. Lung Function in Children and Adolescents. Methods, Reference Values. Basel, Karger, 1987.

23. Bain J, Bishop J, Olinsky A. Evaluation of directed coughing in cystic fibrosis. Br J Dis Chest 1988; 82: 138 148 .

24. Hasani A, Pavia D, Agnew JE, Clarke SW. Regional lung clearance during cough and forced expiration technique (FET): effects of flow and viscoelasticity. Thorax 1994; 49: 557-561.

25. Gosselink HMM. Fysiotherapie ter vermindering van luchtwegobstructie. In: Gosselink HAAM, Cox NJM, Decramer M, Folgering HThM, eds. Fysiotherapie bij CARA. Utrecht, Bunge, 1988; pp. 119-125.

26. Andersen JB, Falk M. Chest physiotherapy in the pediatric age group. Respir Care 1991; 36: 546-554.

27. Hills M, Armitage P. The two-period cross-over clinical trial. Br J Clin Pharmac 1979; 8: 7-20.

28. Lindemann H. Zum Stellenwert der Physiotherapie mit dem VRP 1-Desitin ("Flutter"). Pneumologie 1992; 46: 626-630.

29. Mortensen J, Falk M, Groth S, Jensen C. The effects of postural drainage and positive expiratory pressure physiotherapy on tracheobronchial clearance in cystic fibrosis. Chest 1991; 100: 1350-1357.

30. Lyons E, Chatham K, Campbell IA, Prescott RJ. Evaluation of the Flutter VRP1 device in young adults with cystic fibrosis. Med Sci Res 1993; 21: 101-102.

31. Pryor JA, Webber BA, Hodson ME, Warner JO. The flutter VRP 1 as an adjunct to chest physiotherapy in cystic fibrosis. Respir Med 1994; 88: 677-681.

32. Hofmeyer JL, Webber BA, Hodson ME. Evaluation of positive expiratory pressure as an adjunct to chest physiotherapy in the treatment of cystic fibrosis. Thorax 1986; 41: 951-954.

33. Falk M, Kelstrup M, Andersen JB, et al. Improving the ketchup bottle method with positive expiratory pressure, PEP, in cystic fibrosis. Eur J Respir Dis 1984; 65: 423432. 